\title{
The rectum and bladder doses in intracavitary brachytherapy for cervical cancer
}

\author{
Brahmacharimayum Arunkumar Sharma, PhD, Th. Tomcha Singh, MD
}

Department of Radiotherapy, Regional Institute of Medical Sciences, Imphal, India

\begin{abstract}
Purpose: The aim of this work was to study the International Commission on Radiation Unit and Measurement (ICRU) for rectum (rICRU) and bladder (bICRU) dose behaviors in the treatment of cervical cancer by high dose rate (HDR) intracavitary brachytherapy (ICRT).

Material and methods: A retrospective study was carried out on 38 patients with a total of 38 fractions ( $1^{\text {st }}$ fraction of each patient) of cervical cancer treated with HDR intracavitary brachytherapy in addition to external radiotherapy (EBRT). Manchester system using standard source loading pattern were used in the planning. Normal distributions were tested to the rICUR and bICRU doses for any statistical conclusions.

Results: It was observed that rICUR and bICRU of the population under study is found to show normal distribution at $5 \%$ level of probability with mean dose of $65 \%$ and $61 \%$ and standard deviation of $15 \%$ and $21 \%$, respectively.

Conclusions: The normality behavior of the rectal and bladder doses suggests that not less than $80 \%$ of population (patients) receive rectal and bladder doses less than $80 \%$ of the prescribed dose at point $\mathrm{A}$.
\end{abstract}

Key words: brachytherapy, Manchester system, ICRU rectum/bladder, cervical cancer.

\section{Purpose}

The success of radiation therapy of cervical cancer requires the delivery of high radiation dose directly to the tumor while sparing, to some degree, the surrounding healthy tissues. The curative potential of radiation therapy in the management of cervical cancer is greatly enhanced by the use of intracavitary brachytherapy [1-4]. Brachytherapy is normally used either alone or, more commonly, as a part of multi-modality approach with EBRT, surgery, and/or chemotherapy. In a typical radiotherapy department, about $10-20 \%$ of all radiotherapy patients are treated with brachytherapy [5]. Commonly, brachytherapy is used with EBRT to locally increase the dose to an area at greatest risk for tumor recurrence, such as the original distribution of gross tumor or to the tumor bed at a surgical resection site. The American Brachytherapy Society (ABS) strongly recommends that radiation treatment for cervical cancer (with or without chemotherapy) should include brachytherapy as a component of treatment [6]. The performance of brachytherapy was also critically reviewed by Nag et al. [7].

Rectal and bladder doses in ICRT for cervical cancer are estimated using the International Commission on Radiation Unit and Measurement (ICRU) reference points [8]. Institutions also observed to practice rectal and bladder dose keeping below $80 \%$ of dose at point $\mathrm{A}$ for each fraction [9] in the similar line as recommended by ABS [6].

In this paper, we demonstrated the normality behavior of rectal and bladder dose of all cervical cancer patients undergoing HDR brachytherapy and also critically discussed about the problem of short intrauterine length.

\section{Material and methods}

\section{Patient selection}

Thirty-eight patients of histopathologically confirmed cervical cancer were selected for this retrospective study. We considered only first fraction of each patient. Selected patients were treated with EBRT and high dose rate ICRT between June 2008 and October 2010 in Radiotherapy Department of Regional Institute of Medical Sciences, Imphal, India.

\section{ICRT applicator placement and markers}

Fletcher-suit applicators (Nucletron) were used with ovoid's: half ovoid (15 mm and $20 \mathrm{~mm}$ - diameters) and full ovoid (20 mm - diameter) with tandem angle of $15^{\circ}$, 
$30^{\circ}$ and $45^{\circ}$. Combination of ovoid size and tandem angle were chosen according to patient's anatomy. Packing was done to avoid any shifting or changes in the geometry of the applicators placement and prevent the relocation of rectum and bladder. Foley balloon was inserted and filled with 7 cc of diluted urografin (30\% contrast, $70 \%$ water) and pulled down to be seated on the bladder trigone. Rectal probe have also been inserted in the rectum. All procedures were done without general or spinal anesthesia, after which patients were shifted to simulator room for taking orthogonal films for brachytherapy planning.

As an attempt to analyze the reflection of dose behavior to rectum and bladder in relation to length of uterine tandem loading, 4 patients of the trial were randomly chosen and active source loading for the uterine tandem length was applied for 5.0, 4.3, 3.5, 2.8, 2.0 and $1.5 \mathrm{~cm}$ under one standard source loading system. The measurement of dose for these patients to rectum and bladder was done in relation to dose at point A.

\section{Brachytherapy planning}

Planning was done using Plato Sunrise Treatment Planning system (Nucletron). Dose in the range of 500 to 750 cGy in 3 to 5 fractions were prescribed to point A of Manchester System using standard source loading pattern without optimization. In the planning process, rectal and bladder doses were planned to keep below $80 \%$ of dose to point A for each planned fraction. However, in a few cases either rectum or bladder was beyond $80 \%$ of point $\mathrm{A}$.

\section{Test of normality}

It is one of the most common test for quantitative measures of deviation significance of experimental results from theory. $\chi^{2}$-test for goodness of normality is given by

$$
\chi^{2}=\sum_{\mathrm{i}=1}^{\mathrm{k}}\left\{\mathrm{O}\left(\mathrm{I}_{\mathrm{i}}\right)^{\prime}-\mathrm{I}_{\mathrm{i}}{ }^{\prime}\right\}^{2} / \mathrm{I}_{\mathrm{i}}{ }^{\prime}
$$

where $k$ is the effective number of classes, $O\left(I_{i}\right)^{\prime}$ is the observed frequency and $I_{i}{ }^{\prime}$ is the expected frequency of the

A

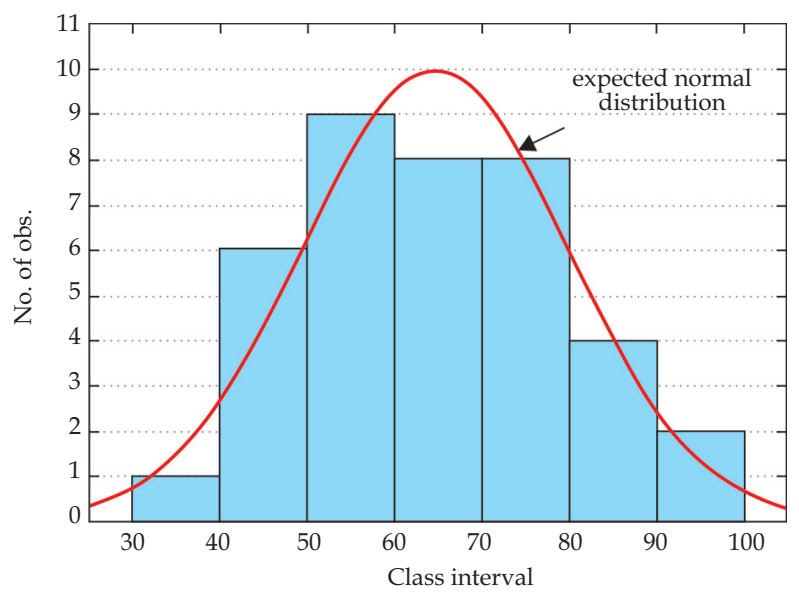

normal distribution. If the distribution follows normality then the value of $\chi^{2}$ is insignificant at least upto the minimum probability level of $1 \%$, which is the lowest critical limit usually assigned to $\chi^{2}$ distribution for acceptability of the hypothesis. Otherwise, $5 \%$ level of probability is conventionally taken as the critical limit for the acceptability of the hypothesis [10-11]. If the hypothesis of normality is accepted, then mean \pm standard deviation will include $66.67 \%$ of the population and mean \pm 2 standard deviation will consist of $95 \%$ of the population.

\section{Results}

Histogram of rectal and bladder dose distribution of 38 patients who underwent HDR intracavitary brachytherapy is shown in Fig. 1A-B along with their expected normal distribution curves. Calculated values of $\chi^{2}$ for normal distribution fitting are found to be 0.94 each with degree of freedom 1. Comparison of these $\chi^{2}$ values with the table values shows that the fits are acceptable at $5 \%$ level of probability. The mean \pm standard deviation of the distribution of rectal and bladder doses are $65 \pm 15 \%$ and $61 \pm 21 \%$, respectively.

In randomly chosen 4 cases in whom differential active loading was done for the uterine tandem, it has been observed that there was a continuously progressive increase in dose to rectum and bladder in relation to point A dose for loading lengths $3 \mathrm{~cm}$ or lesser.

\section{Discussion}

The statistical analysis of these 38 patients suggests that about $87 \%$ and $84 \%$ patients received rectal and bladder doses below $80 \%$ of prescribed dose at point A. Even though sample size was small in number (38), the statistics represents the whole population. So, the most likely rectal dose and bladder dose of this institutional HDR intracavitary brachytherapy practice for ca-cervix are 65 $\pm 15 \%$ and $61 \pm 21 \%$, respectively, of prescribed dose at point A. A recent report [9] shows rICRU and bICRU

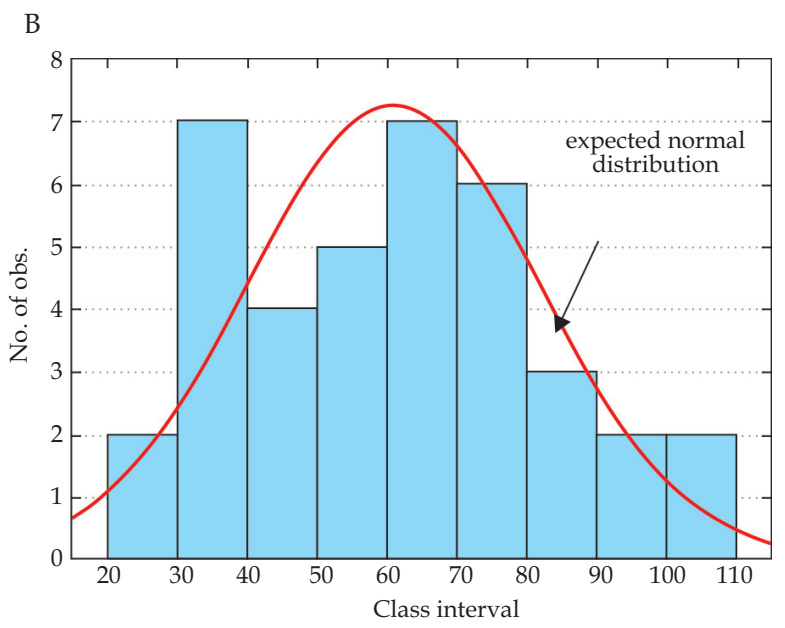

Fig. 1. A) Histogram of rectal doses in percentage of point A (number of observations vs. class interval of dose in $\%$ of point A). B) Histogram of bladder doses in percentage of point A (number of observations vs. class interval of dose in \% of point A) 

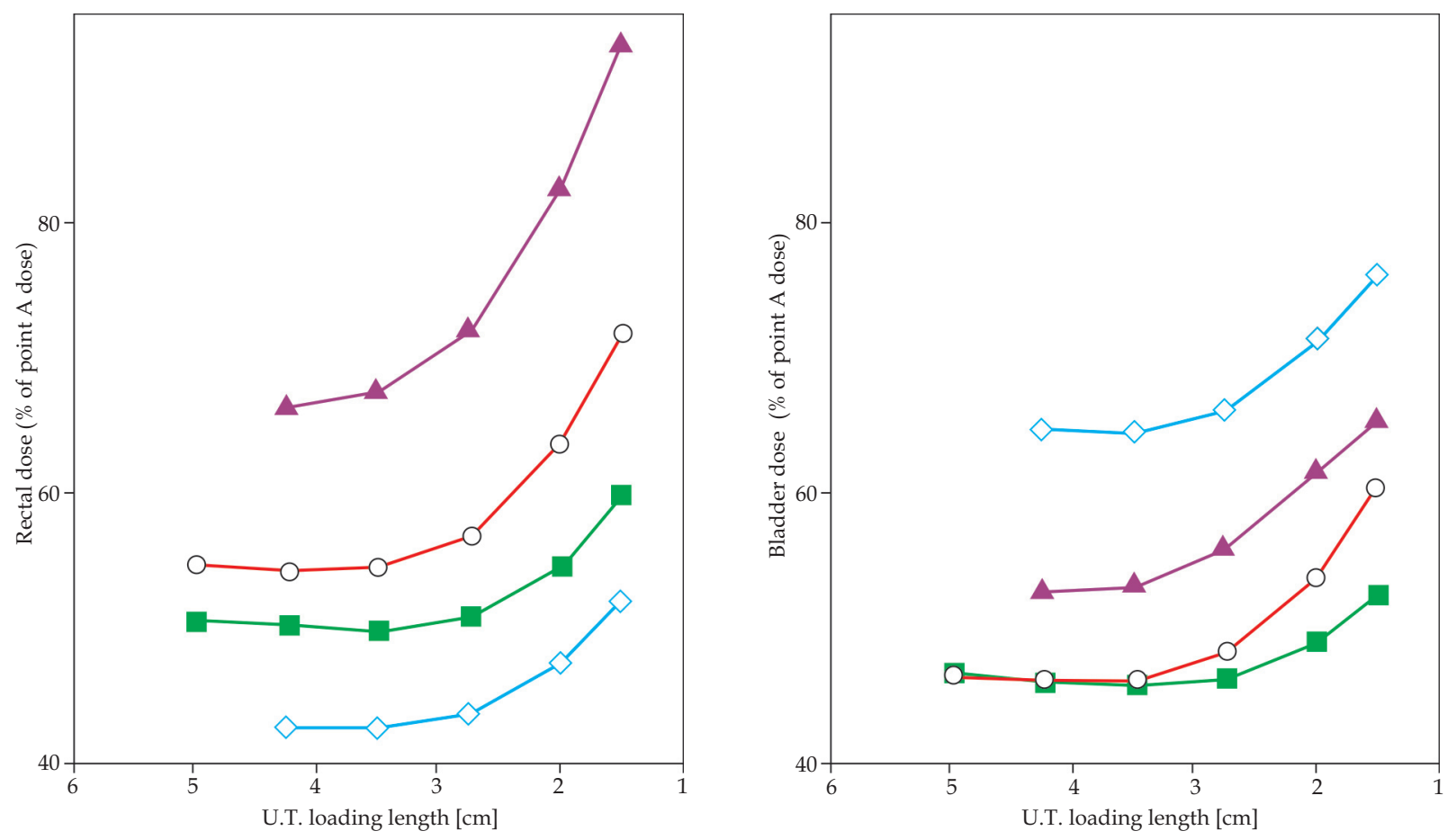

Fig. 2. Graphs showing dose behavior patterns with different uterine tandem (U.T.) loading length; left - rectum and right - bladder

as $72 \pm 6 \%$ and $67 \pm 7 \%$ of point $A$ for 10 patients with a total of 55 fractions. In this case, the number of patients is quite small but the number of fractions is high due to the consideration of other remaining fractions of the same patient which probably reduces the standard deviation.

Table 1 shows the number of patients observed and expected from the study that crosses $80 \%$ of the prescribed dose at point A. Most of these cases have uterine tandem length $\leq 4 \mathrm{~cm}$ (rICRU -4 out of 6 and bICRU -5 out of 7$)$. Shorter intrauterine length have a tendency to increase rectal and bladder dose in Manchester system of defining dose at point $A$ (which is fixed in the geometry) as shown in Fig. 2A-B. For shorter length of intra-uterine $\leq 2 \mathrm{~cm}$, two ovoid technique (source loading to left and right ovoid only), Computerized Tomography based ICBT (volumetric treatment) or interstitial brachytherapy may be useful choice of treatment when rectal and bladder tolerance doses are concerned.

\section{Conclusions}

The normality behavior of the rectal and bladder doses suggests that not less than $80 \%$ of the population (patients) receive rectal and bladder dose less than $80 \%$ of the prescribed dose at point A. The probability of rectal dose below $80 \%$ of prescribed dose at point $\mathrm{A}$ is 0.87 and that of bladder is 0.84 , respectively. Shorter intrauterine length (3 $\mathrm{cm}$ or lesser) have a tendency to increase rectal and bladder dose in Manchester system of defining dose at point $\mathrm{A}$.
Table 1. Number of patients crossing $80 \%$ of prescribed dose at point $A$

\begin{tabular}{lcc} 
& Rectal & Bladder \\
\hline Number of observation (data) & $6 / 38$ & $7 / 38$ \\
\hline Number of expectation (normal distribution) & $5 / 38$ & $6 / 38$
\end{tabular}

\section{References}

1. Lanciano RM, Won M, Coia LR et al. Pretreatment and treatment factors associated with improved outcome in squamous cell carcinoma of the uterine cervix: A final report of the 1973 and 1978 patterns of care studies. Int J Radiat Oncol Biol Phys 1991; 20: 667-676.

2. Montana GS, Fowler WC, Varra MA et al. Carcinoma of the cervix, stage III: Results of radiation therapy. Cancer 1986; 57: 148-154.

3. Perez CA, Breaux S, Madoc-Jones H et al. Radiation therapy alone in the treatment of carcinoma of the uterine cervix: I. Analysis of tumor recurrence. Cancer 1983; 51: 1393-1402.

4. Eifel PJ, Morris M, Oswald MJ. The influence of tumor size and growth habit on outcome of patients with FIGO stage IB squamous cell carcinoma of the uterine cervix. Int J Radiat Oncol Biol Phys 1993; 27: 127-128.

5. IAEA: Podgorsak EB. Radiation oncology physics: a handbook for teachers and students. International Atomic Energy Agency, Vienna 2005.

6. Nag S, Erickson B, Thomadsen B et al. The American Brachytherapy Society recommendations for High-Dose-Rate Brachytherapy for carcinoma of the cervix. Int J Radiat Oncol Biol Phys 2000; 48: 201-211. 
7. Nag S, Dobelbower R, Glasgow G et al. Inter-society standards for the performance of brachytherapy: a joint report from ABS, ACMP an ACRO. Critical Reviews in Oncology/Hematology 2003; 48: 1-17.

8. ICRU. In: Chassagne D, Dutreix A, Almond P et al. ICRU Report No. 38: Dose and Volume specification for reporting intracavitary therapy in gynecology. International Commissioning on Radiation Units and Measurements; Bethesda 1985.

9. Tan YI, Choo BA, Lee KM. 2D to 3D evaluation of organs at Risk doses in intracavitary brachytherapy for cervical cancer. J Contemp Brachyther 2010; 2: 37-43.

10. Spiegel MR. Schaum's outline of Theory and problems of Probability and Statistics SI (Metric). McGraw-Hill Inc, Singapore 1980.

11. Everitt BS. The analysis of contingency tables. Chapman and Hall Ltd., London 1977. 